\title{
Role of Symmetry in an Asymmetric System
}

\author{
Magdalena Kubik \\ Physics Department, University of Warsaw, Poland.
}

\begin{abstract}
The aim of the study is to present features of a complex system and find the relations making the symmetry gain its sense in asymmetric wold. The study prepared in the form of a mental experiment shows that a system can exist in two states and two orientations and the mechanisms described in the study transform the system. The mechanisms causing the transformations are also presented as well as the role of symmetry which leads to the system's asymmetry.
\end{abstract}

Keywords: Symmetry, asymmetry in systems, complex systems, interdisciplinary study

The study's objective is to present the complex system features and find the relations which make the symmetry gain its sense in asymmetric world. The study contains a description of a mental experiment inspired by Richard Dawkins' thought taken from the preface to the second edition of his book The Selfish Gene. With this experiment is used to show that a system can exist in two states and two orientations and the mechanisms described in the experiment transform the system. The mechanisms causing the transformations are also presented as well as the role of symmetry which leads to the system's asymmetry. The "Necker cube" metaphor is used here, which was also written about in the preface to Richard Dawkins' book.

The properties of "Necker cube" are the reason of a mental travel between two extremes while looking at it. However if a person observes a complex system in the same way as the "Necker cube" the effect of perception is diverted, which means that the person does not notice the extremes. An example used for the mental experiment is a group of people drinking juice. The experimental group consists of three subgroups: right-handed people, left-handed people and people who use both hands equally. There is an assumption in the experiment that the

Corresponding author: Magdalena Kubik, Master of Finance and Accounting, research field: interdisciplinary study of systems. E-mail: kubik.magdalena@wp.pl. right hand of a both hands user is symmetrical to the right and the left hand of a both hands user and the left hand of a left hand user. Consequently, the left hand of a both hands user is symmetrical to the left and the right hand of a both hands user and the right hand of a right hand user. In the experiment there is a certain amount of juice and a group of people holding glasses. The juice is poured into glasses and they start drinking. The transformation process is being observed.

The above described experimental groups are sitting by the table. Each group member is holding a glass with the juice in it and wants to have a drink. Regardless the fact that the experimental group is divided into three subgroups each person is holding the glass with the right or left hand. Observing this system it is difficult to imagine the effect as in the case of the "Necker cube". The hands rising glasses are seen, however all the right hands cannot be seen at the same time or all the left hands at the same time. It is difficult to notice the number of the experimental group members drinking holding the glasses with their right or left hand. In the opposition to the "Necker cube" it is difficult to notice the extremes. In the case of no disturbance occurring, like spilling the juice on the floor, it is difficult to notice anything more but the hands holding glasses. Only the process of drinking 
can be observed. In the repeated experiment the subgroup of both hands users becomes here the reason why the system oscillates between two symmetrical extremes, which are the people drinking with glasses in their right hands and the people drinking with glasses in their left hands.

The reason of the oscillation between the two extremes is the factors which start the mechanisms influencing the transformations in the extremes.

Another aspect of the mental experiment is in the case when the disturbance occurs. The disturbance is the situation when some members of the experimental group accidentally spill the juice on the floor instead of drinking it. In case of the disturbance the two symmetrical extremes (the people drinking with glasses in their right hands and the people drinking with glasses in their left hands) between which the system oscillates are not visible, however the disturbance is noticeable. The factor which appears there is the feeling of risk that the juice may be spilt and the fear starts some mechanisms. In order to prevent the risk some of the both hands users will change hands holding the glasses. Some of the experimental group members who have other options will use them. Transformations in the system are started by neutral experimental group members, as only the both hands users can change hands. This is the case when the disturbance starts the symmetric extreme and that is why the symmetric system transformation is not visible. The number of people drinking with glasses in their right hands has changed and the number of people drinking with glasses in their left hands has also changed. One of the symmetrical extremes started the disturbance and it caused the system transformation. The symmetrical transformation of the system causes that the changes are not visible.

In the case of the disturbance described above part of the juice has been spilt. It means that not all the experimental group members had a drink and this situation leads to an asymmetry in the system. Because of the transformations some of the people in the experimental group spilt the juice and did not drink at all. Some of the people had a drink and are not thirsty any more. Some of the people drank just the juice which had not been spilt before. In the experimental group there are people who are not thirsty, those who are thirsty, and those who drank a bit and can have a drink but are not thirsty; however the system still oscillates between the two extremes. The extremes are the people who will drink and those who will not drink. There is symmetry between the people drinking with glasses in their right hands and the people drinking with glasses in their left hands and there is also an asymmetry between those who will drink and those who will not drink. The occurring asymmetry influences the scope of the symmetry. The group of drinking people increases and the group of not drinking people decreases. The scope of the active symmetric mechanisms decreased. The symmetric mechanisms work in asymmetric extremes which contain people who will drink and those who will not drink. After a certain time those mechanisms start the transformations in asymmetric extremes and it causes oscillation in the system.

Considering above mentioned asymmetric extremes (those who will drink and those who will not drink) it can be concluded that in the group of people who will drink are those who are thirsty because they did not drink anything and those who want to drink as they drank just a bit left from not spilling. In the group of people who will not drink are those who are not thirsty because they drank all they had had in their glasses and those who want to drink as they drank just a bit left from not spilling. The people who drank a bit will want to drink some more and the group of people who want to drink will increase or there is a possibility that they will resist from drinking trying to avoid spilling the juice and as a result the group of 
people who do not want to drink will increase. There will be another asymmetric factor: the fear from the risk of spilling the juice and the wish to drink. This factor will cause the symmetric mechanisms in asymmetric extremes. In the group of people who want to drink there will appear a symmetric mechanism which will cause a transformation in asymmetric extremes and the number of people who will drink will increase or the number of people who will not drink will increase. After a time people who drank just a bit left from not spilling will become thirsty again and the people who were not thirsty will become the people who want to drink again. There will appear symmetric transformation of the asymmetric extremes and as a result the number of people who will drink for another time. It is a symmetric transformation of the extreme because the people who drink use the juice to produce energy and excrete. Those people who do not drink, because they have stored the juice drank before only excrete. In both cases however all people in both groups will wish to drink after a time. They will all make the asymmetric extremes to expand again.

The people who drink and those who do not drink are two asymmetric extremes, which appeared because of the symmetric factor which is the fear from the risk of spilling the juice and the wish to drink. The factor started the symmetric mechanisms. Those mechanisms made the symmetric factor appear. The factor is the fear from the risk of spilling the juice and the wish to drink. The mechanism started symmetric mechanisms which made the asymmetric extremes appear. The extremes are the group of people who will drink and the group of people who will not drink. Another asymmetric factor which appears here is the expense and benefit. This factor starts another symmetric mechanism influencing two asymmetric extremes to work in the system. The extremes are the group of people who drink and the group of people who do not drink.
The people who have just had a drink lose and gain at the same time. They excrete and they also use the drunken juice to produce energy. They want to drink and they will expand the group of those who will not drink. By the system transformation they will move to the extreme of people who will drink and then by another system transformation to the extreme of people who drink. The people who do not drink excrete the drunken juice and they also have the juice stored. They also can have another drink and as those who are not thirsty any more they can expand the extreme of those who will not drink. After another system transformation they will move to the extreme of people who will drink. Because of the excreting process the same people will wish to drink and after the system transformation they will move from the extreme of those who will drink to the extreme of those who drink.

The factors, mechanisms and transformations described in the presented mental experiment can be used to explain the paradox mentioned by Richard Dawkins in the preface to the second edition of his book The Selfish Gene:

"In the dozen years since The Selfish Gene was published its central message has become textbook orthodoxy. This is paradoxical, but not in the obvious way. It is not one of those books that was reviled as revolutionary when published, then steadily won converts until it ended up so orthodox that we now wonder what the fuss was about. Quite the contrary. From the outset the reviews were gratifyingly favorable and it was not seen, initially, as a controversial book. Its reputation for contentiousness took years to grow until, by now, it is widely regarded as a work of radical extremism. But over the very same years as the book's reputation for extremism has escalated, its actual content has seemed less and less extreme, more and more the common currency." 1

\footnotetext{
${ }^{1}$ R. Dawkins, The Selfish Gene, Oxford University Press, Oxford 1990
} 


\section{SCHEME:}

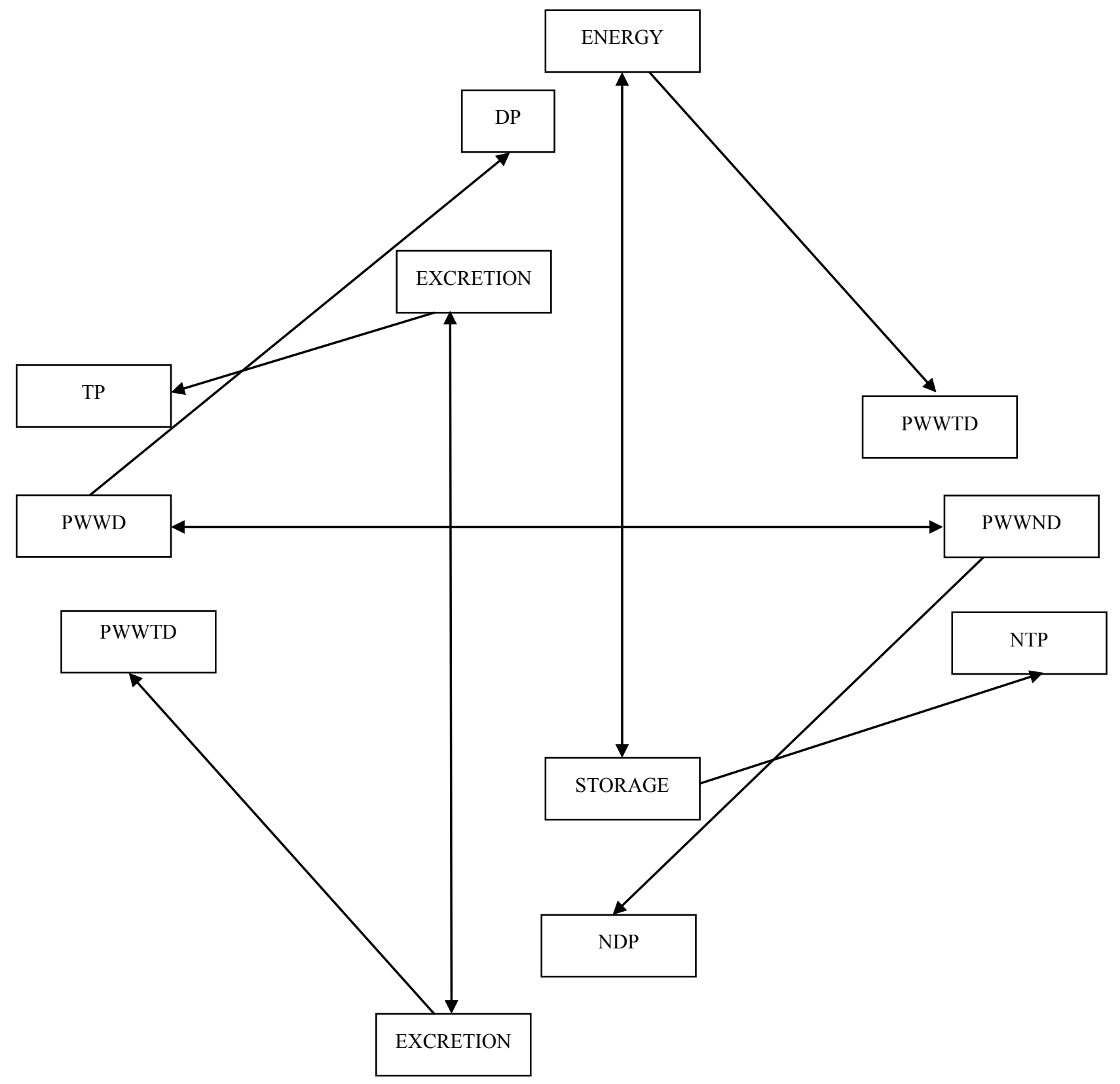

\section{Symbols:}

DP - drinking people

NDP - not drinking people

PWWD - people who will drink

PWWND - people who will not drink

TP - thirsty people

NTP - not thirsty people

PWWTD - people who want to drink 